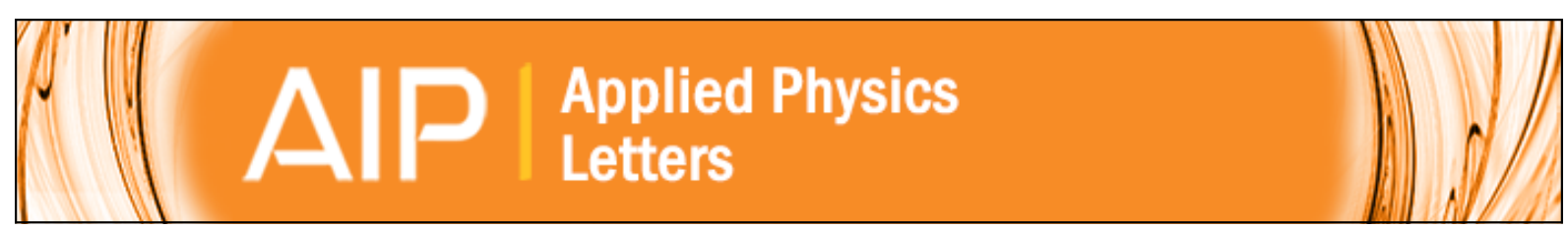

\title{
Stretch-induced softening of bending rigidity in graphene
}

Xinghua Shi, Bo Peng, Nicola M. Pugno, and Huajian Gao

Citation: Applied Physics Letters 100, 191913 (2012); doi: 10.1063/1.4716024

View online: http://dx.doi.org/10.1063/1.4716024

View Table of Contents: http://scitation.aip.org/content/aip/journal/apl/100/19?ver=pdfcov

Published by the AIP Publishing

Advertisement:

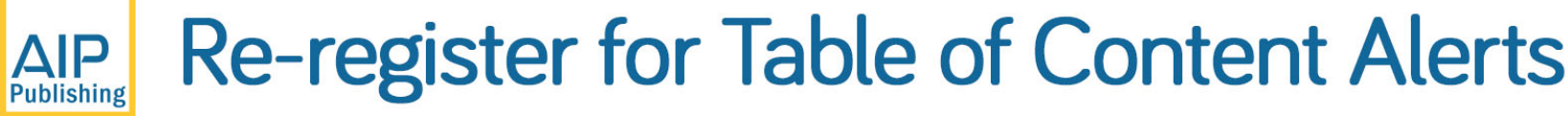

Create a profile.

Sign up today! 


\title{
Stretch-induced softening of bending rigidity in graphene
}

\author{
Xinghua Shi, ${ }^{1, a)}$ Bo Peng, ${ }^{2}$ Nicola M. Pugno, ${ }^{3}$ and Huajian Gao ${ }^{4, b}$ \\ ${ }^{1}$ State Key Laboratory of Nonlinear Mechanics, Institute of Mechanics, Chinese Academy of Sciences, \\ Beijing 100190, China \\ ${ }^{2}$ Department of Physics, Renmin University of China, Beijing 100872, China \\ ${ }^{3}$ Laboratory of Bio-Inspired Nanomechanics “Giuseppe Maria Pugno,” Department of Structural Engineering, \\ Politecnico di Torino, Corso Duca degli Abruzzi 24, 10129 Torino, Italy \\ ${ }^{4}$ School of Engineering, Brown University, 610 Barus \& Holley, 182 Hope Street, Providence, Rhode Island \\ 02912, USA
}

(Received 4 February 2012; accepted 26 April 2012; published online 10 May 2012; publisher error corrected 18 May 2012)

First principle calculations are performed to show that the bending rigidity of graphene can be softened considerably with in-plane stretching. This phenomenon can be attributed to stretch-induced loosening of atomic packing and should be of fundamental significance for graphene-based structures and devices. (C) 2012 American Institute of Physics. [http://dx.doi.org/10.1063/1.4716024]

Graphene consists of a single atomic layer of carbon atoms densely packed in a hexagonal crystal lattice. ${ }^{1}$ Considerable attention has been focused on the stability of the twodimensional lattice of graphene ${ }^{2}$ as well as its exceptional low-dimensional electronic, thermal, optical, and mechanical properties $^{3-5}$ that can be potentially utilized in nextgeneration durable, reliable, foldable, and stretchable electronic and nanoelectromechanical devices. ${ }^{6,7}$ In particular, the behaviors of graphene under coupled stretching and bending are of fundamental significance for the study of graphene-based structures and devices. ${ }^{8,9}$ It is known that wrinkling of a thin elastic sheet depends strongly on both the bending stiffness and stretching, ${ }^{10}$ and controllable ripples in suspended graphene have indeed been created via in-plane stretching. ${ }^{11}$

The behavior of graphene under uniaxial stretching has been studied by molecular mechanics simulations ${ }^{12-15}$ and first-principle calculations. ${ }^{16-21}$ The bending stiffness of graphene has also been studied through theoretical ${ }^{12,13,22-25}$ and first-principle calculations. ${ }^{26-28}$ However, the coupling between bending and stretching, in particular the bending stiffness of graphene in a stretched state, has not been reported in the literature. While it can be anticipated that the bending rigidity of graphene could be affected by in-plane stretching, a quantitative study must be conducted to gain a full understanding of the issue.

The bending stiffness of graphene in a free state has been studied in the literature. ${ }^{13,22,24-28}$ In standard approach, fully relaxed graphene sheets are rolled into a set of cylindrical nanotubes with different radii. The strain energy density of these tubes can be expressed as $U=\frac{D}{2 R^{2}}$, where $D$ is the bending stiffness of graphene and $R$ is the tube radius. By plotting the calculated values of $U$ as a function of $1 / R^{2}$, the bending stiffness of graphene is determined through a linear fitting process. Here, we adopt the same strategy to calculate the bending stiffness of pre-stretched graphene sheets. In this case, the strain energy density is $U=\frac{D}{2 R^{2}}+U_{\text {stretch }}$, where $U_{\text {stretch }}$ is a reference energy term with no effect on the fitting

\footnotetext{
${ }^{\text {a)} E-m a i l: s h i x h @ i m e c h . a c . c n . ~}$

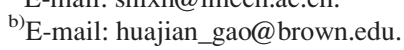

process. According to the direction of applied stretch, the system under investigation can be divided in three categories: (1) uniaxial stretch of graphene in the armchair direction (Fig. 1(a), y-direction); (2) uniaxial stretch of graphene in the zigzag direction (Fig. 1(a), $x$-direction); and (3) equibiaxial stretch of graphene.

Density functional theory (DFT) calculations were performed using the Vienna ab initio simulation (VASP) package $^{29,30}$ with the projector augmented wave (PAW) method $^{31}$ and the Perdew-Burke-Ernzerhof (PBE) generalized gradient approximation (GGA) for exchange and correlation. A plane-wave basis set with a kinetic energy cutoff of $550 \mathrm{eV}$ and a Monkhorst-Pack $k$-point mesh of $21 \times 21 \times 1$ are used. Atoms are relaxed using a conjugate gradient algorithm until the interatomic forces are less than $0.1 \mathrm{eV} / \mathrm{nm}$. The structural relaxation and stress-strain calculations are performed in a four-atom unit cell shown in Fig. 1(a). The size of the periodic box in the $z$-direction is set at $1 \mathrm{~nm}$ to avoid interactions between the graphene sheet and its periodic images. In the absence of stretching, the C-C bond length is calculated to be $d=0.14217 \mathrm{~nm}$, in good agreement with the experimental value of $0.14209 \mathrm{~nm}^{32}$ As the graphene is stretched, for instance in the $x$-direction, one of the lattice length is increased from $a_{0}$ to $a$ and then kept fixed (Fig. 1(a)). The other lattice length $b$ is varied until the total energy of the system is minimized. The obtained lattice constants $a, b$, and $d$ are then used to construct nanotubes in a strained state. For biaxial stretching, both $a$ and $b$ are increased with the same strain, followed by structural relaxation with the lattice constants fixed.

With the unit cell structure of graphene at specific strains obtained, we construct a set of graphene sheets and roll them into nanotubes. Depending on the stretching and rolling directions, we label the calculated bending stiffness as $D_{j}^{i}$ where $i$ denotes the stretching direction and $j$ is the direction of rolling, as shown in Fig. 2. For instance, $D_{z i g}^{a r m}$ indicates the bending stiffness of graphene when stretched in the armchair direction but rolled about the zigzag direction. DFT calculations are then performed to optimize the structure using a similar method as described above except that the Monkhorst-Pack $k$-point mesh is set at $1 \times 1 \times 20$. 
(a)

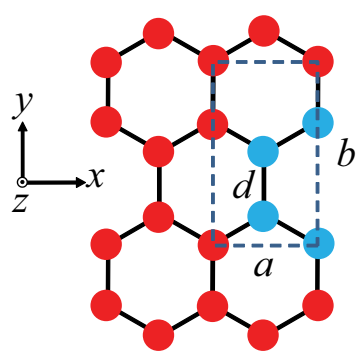

(c)

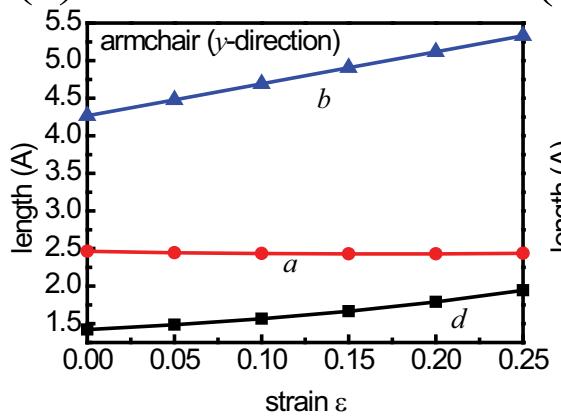

(d)

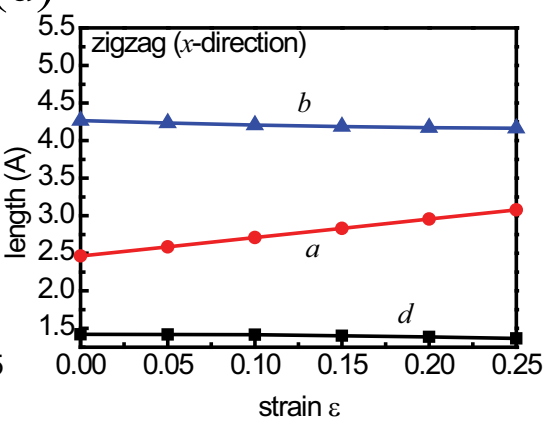

(b)

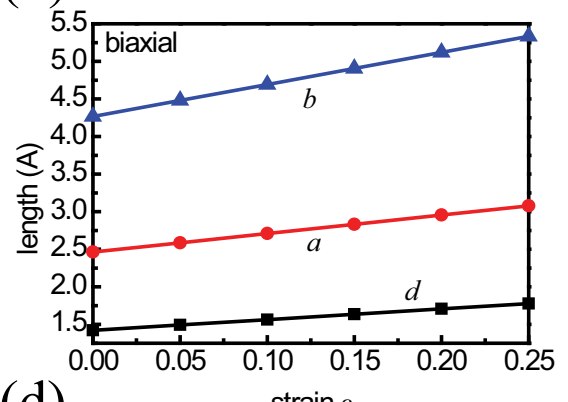

FIG. 1. Deformation in a unit cell of graphene. (a) The four-atom unit cell used in stress-strain calculations. Three parameters, $a, b$, and $d$, are used to describe the deformation. Variations of the parameters as the unit cell is (b) stretched equibiaxially, or uniaxially in the (c) armchair or (d) zigzag directions.

\section{(a)}

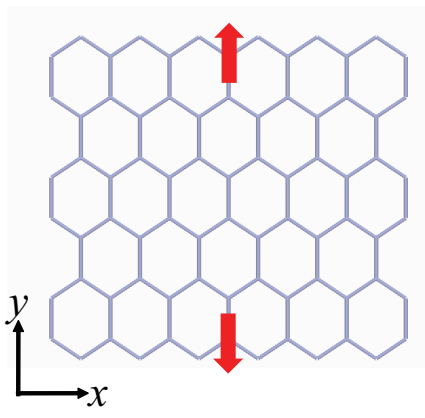

(b)

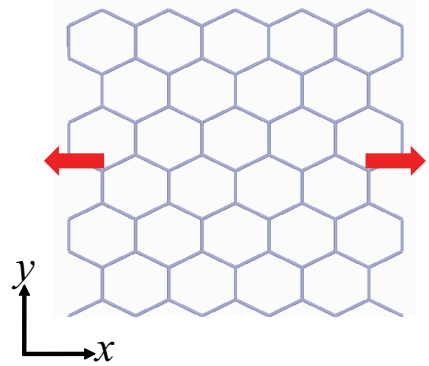

(c)

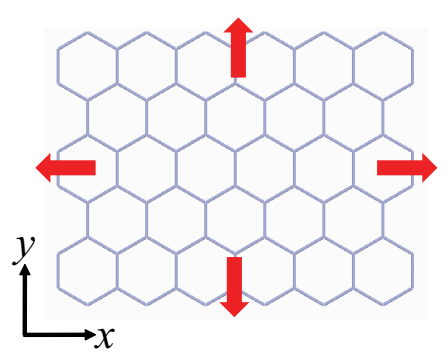

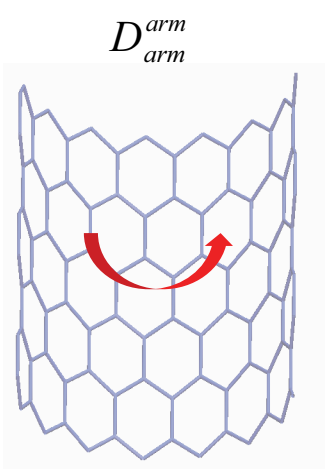
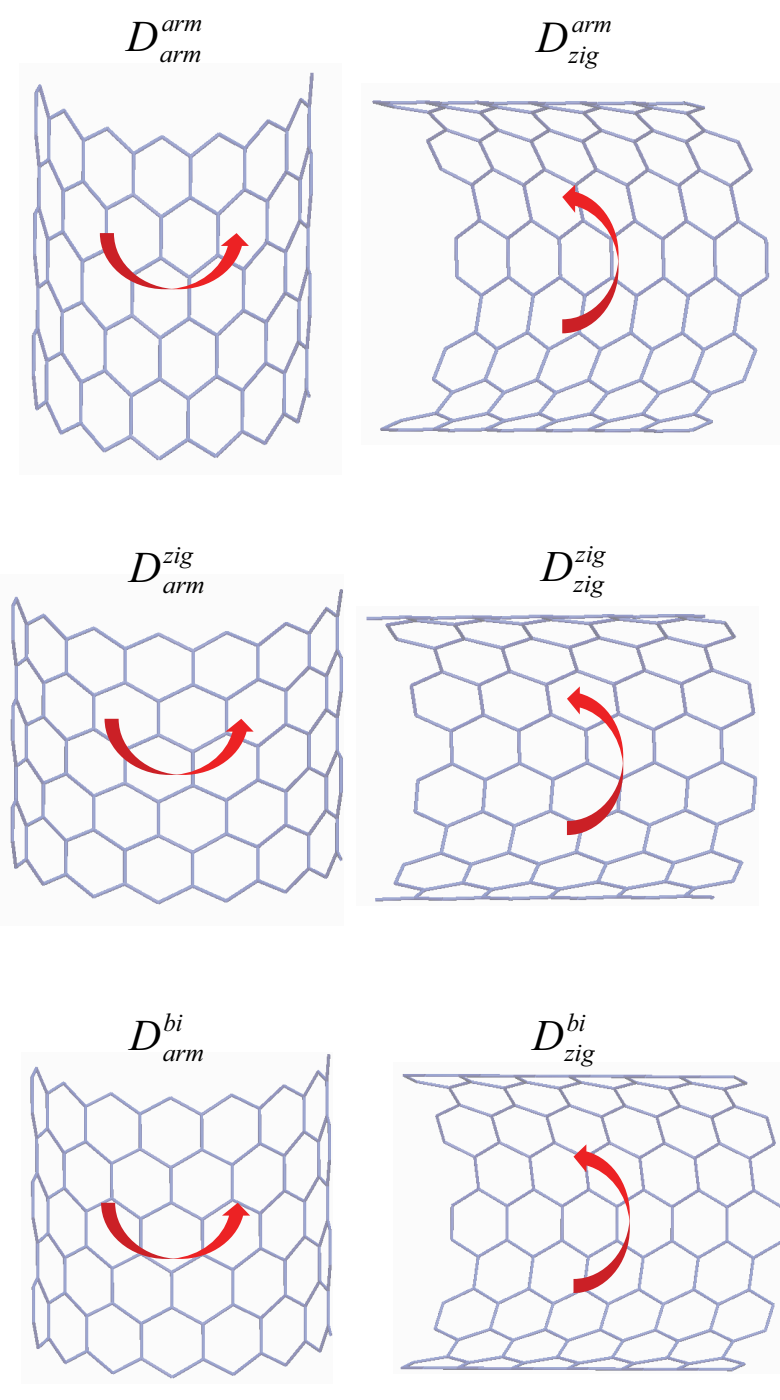

FIG. 2. Schematic of stretching and rolling of graphene sheets: (a) stretching in the armchair direction and rolling about the armchair and zigzag directions; (b) stretching in the zigzag direction and rolling about the armchair and zigzag directions; and (c) equibiaxial stretching and rolling about the armchair and zigzag directions. 
(a)
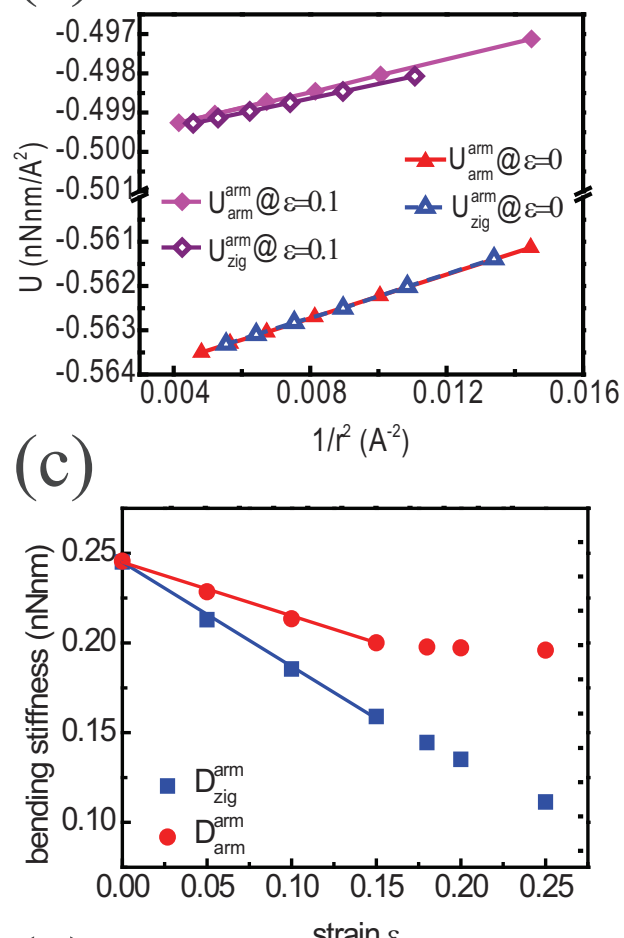

(e)

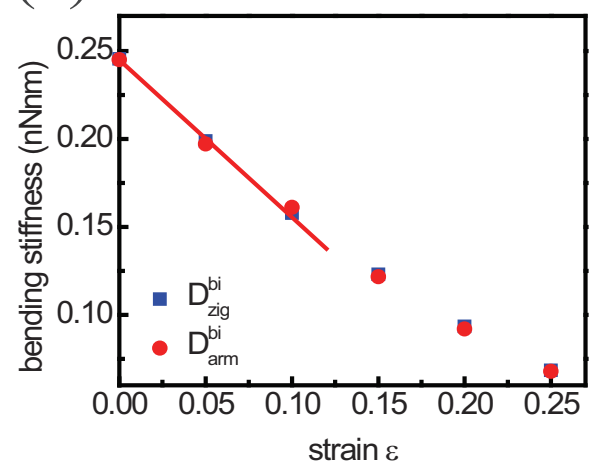

(b)

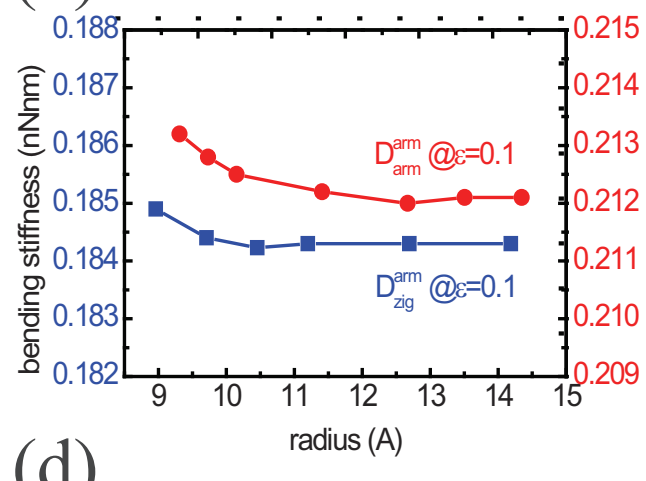

(d)

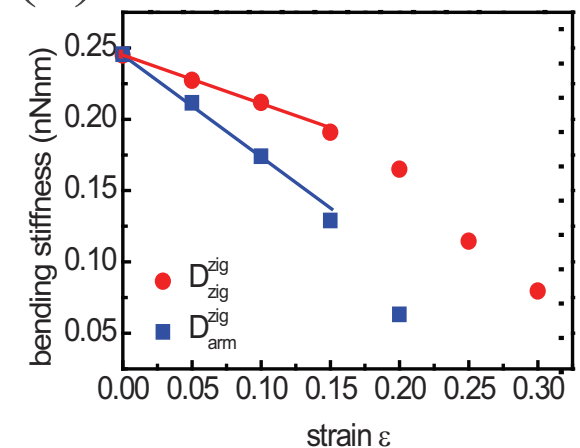

(f)

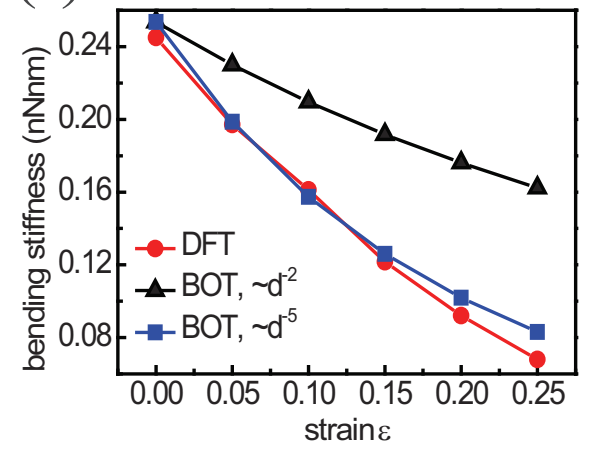

FIG. 3. Stretch induced softening of bending resistance in graphene. (a) Strain energy density profiles for nanotubes made of pre-stretched graphene sheets against the inverse square of the tube radius. The solid and open squares are DFT results for $U_{a r m}^{a r m}$ and $U_{z i g}^{a r m}$ when $\varepsilon=0.1$, while the solid and open triangles are DFT results for $U_{\text {arm }}^{\text {arm }}$ and $U_{z i g}^{\text {arm }}$ when $\varepsilon=0$. The corresponding lines are linear fittings whose slope is equal to half of the bending stiffness. (b) The bending stiffnesses of graphene $D_{z i g}^{a r m}$ and $D_{a r m}^{a r m}$ as a function of the tube radius when $\varepsilon=0.1$ (c-e) The bending stiffness of graphene as a function of the stretching strain. For $\varepsilon<0.15$, the stiffness-strain relation can be approximated by a linear function $D_{j}^{i}=D_{0}-\lambda_{j}^{i} \varepsilon$, where $D_{0}=0.245 \mathrm{nN} \cdot \mathrm{nm}$, $\lambda_{\text {zig }}^{\text {arm }}=0.569 \mathrm{nN} \cdot \mathrm{nm}, \lambda_{\text {arm }}^{\text {arm }}=0.299 \mathrm{nN} \cdot \mathrm{nm}$, $\lambda_{\text {arm }}^{z i g}=0.714 \mathrm{nN} \cdot \mathrm{nm}, \quad \lambda_{z i g}^{z i g}=0.332 \mathrm{nN} \cdot \mathrm{nm}$, and $\lambda_{\text {arm }}^{b i}=\lambda_{z i g}^{b i}=0.877 \mathrm{nN} \cdot \mathrm{nm}$. (f) Comparison between the bending stiffness of graphene obtained from DFT (red) calculations and that from bond orbital theory with $d^{-2}$ (black) and $d^{-5}$ (blue) scaling laws.
During optimization, the radius of CNT is fixed to eliminate the strain induced by rolling, as well as to preserve the prestrain in case the graphene sheet is pre-stretched along the rolling direction. ${ }^{22}$

The lattice constants $a, b$ and the C-C bond length $d$ in the armchair direction are shown in Figs. 1(b)-1(d) for different magnitudes of stretching strain $\varepsilon=\left(a-a_{0}\right) / a_{0}$ or $\varepsilon=\left(b-b_{0}\right) / b_{0}$. Under equibiaxial stretching, all the lattice constants increase with strain (Fig. 1(b)), resulting in a uniform expansion of the hexagonal structure of graphene. Under uniaxial stretching, however, the lattice constant in the direction perpendicular to stretching varies only slightly, and the deformed lattice of graphene is no longer a perfect hexagon (Figs. 1(c) and 1(d)).

The strain energy density profiles for nanotubes rolled up from a set of pre-stretched graphene sheets are plotted against the inverse square of the tube radii in Fig. 3(a). The slope of these profiles gives the bending stiffness of stretched graphene. At very small strains $\varepsilon \approx 0$, the energy profiles (red and blue triangles) for different rolling directions match well with each other, indicating that the bending stiffness of graphene is independent of the rolling direction in the ab- sence of stretch. However, at $\varepsilon=0.1$, the energy profiles (pink and purple rhombuses) become substantially dependent on the direction of rolling, indicating rising anisotropy under uniaxial stretching. Fig. 3(b) shows that the calculated bending stiffness converges to a constant value once the tube radius exceeds about $1 \mathrm{~nm}$, although the estimates based on smaller tubes can be slightly (up to $0.5 \%$ ) higher than the converged value. In this way, the bending stiffness of graphene is extracted from the converged values as in Fig. 3(b) for different stretching and rolling directions, with results summarized in Figs. 3(c)-3(e). It is clearly seen that the bending stiffness shows significant softening at increasing stretching strain. Such softening obviously depends on the rolling direction under uniaxial stretching. Fig. 3(c) shows that, when stretched in the armchair direction, the bending resistance of graphene softens faster along the zigzag direction than along the armchair direction; the opposite is true when stretched in the zigzag direction (Fig. 3(d)). Under equibiaxial stretching, the bending resistance softens but remains isotropic (Fig. 3(e)). In all cases, the bending stiffness $D_{j}^{i}$ decreases linearly with rising strain $\varepsilon$ in the range $0 \leq \varepsilon<0.15$, and can be fitted to an approximate formula 
(a)
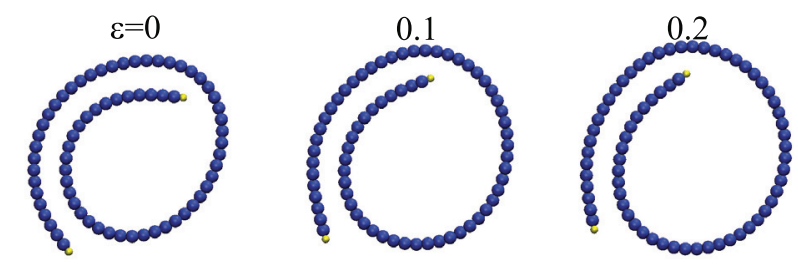

(b)
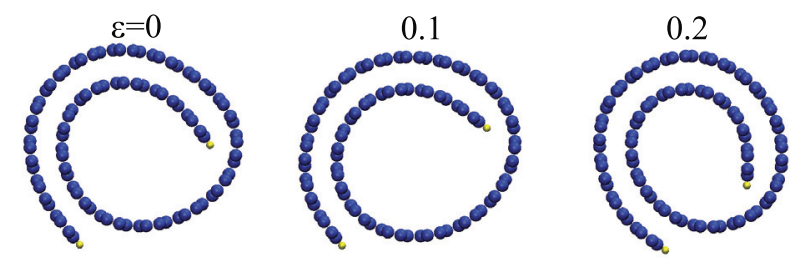

(c)

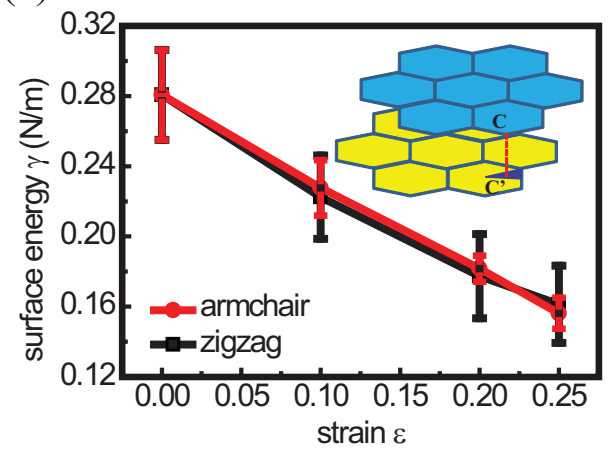

(d)

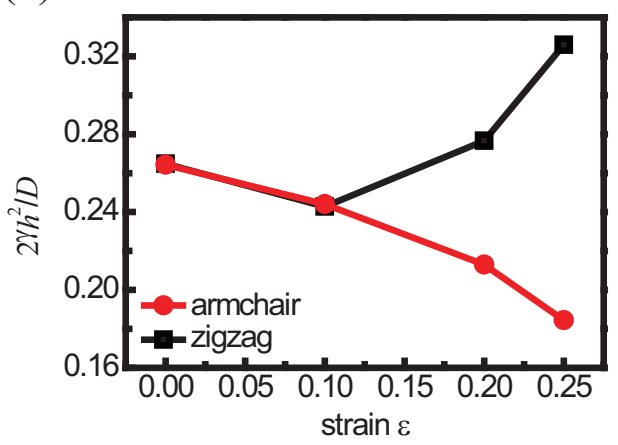

FIG. 4. The equilibrium structures of (a) zigzag and (b) armchair CNS subjected to axial stretching at strain levels of $\varepsilon=0,0.1,0.2$. The blue atoms are carbon and yellow ones are hydrogen. (c) Mean surface energy of armchair and zigzag CNSs as a function of the corresponding axial stretching strain. Inset shows a schematic of stacking between two graphene layers where atom $\mathrm{C}$ in the upper layer projects onto position C' within the lower layer. (d) Variation of the dimensionless parameter $\beta=2 \gamma h^{2} / D$ with strain as graphene is stretched in the armchair (red) and zigzag (black) directions.
$D_{j}^{i}=D_{0}-\lambda_{j}^{i} \varepsilon$ with $D_{0}=0.245 \mathrm{nN} \cdot \mathrm{nm}$. The best fitted values of the softening coefficients for $\varepsilon<0.15$ are $\lambda_{z i g}^{\text {arm }}=0.569 \mathrm{nN} \cdot \mathrm{nm}$, $\lambda_{\text {arm }}^{\text {arm }}=0.299 \mathrm{nN} \cdot \mathrm{nm}, \lambda_{\text {arm }}^{\text {zig }}=0.714 \mathrm{nN} \cdot \mathrm{nm}, \lambda_{\text {zig }}^{\text {tig }}=0.332 \mathrm{nN} \cdot \mathrm{nm}$, and $\lambda_{\text {arm }}^{b i}=\lambda_{\text {zig }}^{b i}=0.877 \mathrm{nN} \cdot \mathrm{nm}$.

For strain $\varepsilon>0.15$, the softening of bending stiffness no longer obeys the linear softening equation. In this range, $D_{\text {arm }}^{\text {arm }}$ remains almost constant. This peculiar behavior could be understood from the packing of atoms along the rolling direction. It can be seen from Fig. 1(c) that the lattice $a$ only varies mildly with the strain, indicating the stretching along the armchair direction does not increase the spacing between atoms along the zigzag direction. The close-packing of atoms along the rolling direction thus prevents the softening of bending stiffness in this configuration. For other cases, the atomic spacing along the rolling direction is usually enlarged by the applied strain, which then leads to the softening of bending stiffness.

One can also use the bond orbital theory (BOT) to understand why the stretching strain can substantially influence the bending stiffness of graphene. From the $\pi$-orbital misalignment between adjacent pairs of carbon atoms in a free standing graphene sheet, it has been estimated that $D=-\frac{2}{3} V_{p p \pi}{ }^{28}$ where $V_{p p \pi}=-0.63 \frac{\hbar^{2}}{m d^{2}}$ is a universal parameter, $\hbar$ is the reduced Planck constant, $m$ is the mass of electron, and $d$ is the interatomic distance. It can be immediately seen that the bending stiffness of graphene decreases with $d$ as $D \sim d^{-2}$. However, the $d^{-2}$ scaling law is only applicable for atoms around the ground state. In a strained state where $d$ deviates from its equilibrium value, the universal parameter usually scales as $d^{-n}$ where $n>2$. For instance, $V_{p p \pi} \sim d^{-4.51}$ for silicon and $V_{p p \pi} \sim d^{-4.76}$ for germanium. ${ }^{33}$ We have applied the bond orbital theory in estimating the bending rigidity of graphene under biaxial stretching with $V_{p p \pi} \sim d^{-2}$ or $d^{-5}$. Figure 3(f) shows that the $V_{p p \pi} \sim d^{-5}$ case agrees with our DFT calculations.

As an example of demonstrating the effect of stretchinduced softening of bending rigidity in graphene, we consider the core size variation in pre-stretched carbon nanoscrolls (CNSs), which is known to depend on both the bending stiffness and surface energy of graphene. ${ }^{34}$ In this analysis, one armchair CNS and one zigzag CNS are first stretched axially to specific strains. DFT calculations are then performed to optimize the geometry with the Monkhorst-Pack $k$-point mesh set at $1 \times 1 \times 20$. Additional van der Waals energy term is added to describe the long range interactions. ${ }^{35}$

Figs. 4(a) and 4(b) show that the zigzag and armchair CNSs exhibit different core sizes at various stretching strains $\varepsilon=0,0.1,0.2$. For the zigzag CNS, the core size increases monotonically with strain (Fig. 4(a)). However, for the armchair CNS, the core size is seen to increase with strain for $\varepsilon=0.1$ but then decrease with strain for $\varepsilon=0.2$ (Fig. 4(b)). To elucidate the mechanism inducing such complicated change in core size, we recall the equation governing the core size of CNS from Ref. 34, $\frac{2 \gamma h^{2}}{D}=\frac{h}{r_{0}}-\frac{h}{\sqrt{r_{0}^{2}+B h / \pi}}$, where $\gamma$ is the surface energy of graphene, $h=0.34 \mathrm{~nm}$ is the interlayer spacing between graphene layers, $B$ is the total length of graphene sheet that forms the CNS, and $r_{0}$ is the core size. It is seen that the core size is influenced by the dimensionless parameter $\beta \equiv \frac{2 \gamma h^{2}}{D}$, which depends on both the bending stiffness and surface energy. 
Additional DFT calculations are conducted to determine the surface energy of graphene under stretching. In contrast to graphite in which the layers of graphene follow the $A B$ stacking, the layers of graphene in CNS have arbitrary stacking as indicated in Fig. 4(c), where the carbon atom $\mathrm{C}$ in the upper layer may project onto any position $\mathrm{C}^{\prime}$ within the blue triangle in the lower layer. We selected a total of 21 different stacking forms and conducted DFT calculations for the mean surface energy shown in Fig. 4(c). It is seen that the surface energy decreases linearly with strain under uniaxial stretching in both directions. With the obtained mean surface energy, we calculate the dimensionless parameter $\beta=\frac{2 \gamma h^{2}}{D}$ at various strains, where the value $D$ is from Fig. 3. Fig. 4(d) shows that, as a zigzag CNS is stretched along the armchair direction, the value of $\beta$ keeps on decreasing, suggesting that the core size of CNS should increase with strain monotonically in this configuration. For an armchair CNS, however, $\beta$ first decreases and then increases with strain, indicating that the core size of CNS should first increase and then decrease with strain. These results are fully consistent with the observed variations in core size of CNSs shown in Figs. 4(a) and 4(b).

In summary, we have studied the softening of bending resistance of graphene under stretching. It is found that the bending stiffness depends strongly on the stretching strain, stretching direction, and rolling direction. Under uniaxial stretching, the structure of graphene becomes anisotropic, leading to a strong dependence of bending stiffness on the stretching and rolling directions. The softening is mainly attributed to the stretch induced loosening of atomic packing along the rolling direction, which is demonstrated in our calculations of the electronic structure. In the special case when graphene is first stretched along and then bent about the armchair direction, it shows a non-softening behavior as the strain increases beyond a critical value. The phenomenon of stretch-induced softening of bending rigidity in graphene has been demonstrated by considering the core size variation of carbon nanoscrolls under axial stretching. As the bending stiffness directly affects the morphology of graphene and can subsequently influence its electronic properties, the results derived in this work provides a quantitative framework for the modeling and design of graphene-based nanoelectromechanical devices.

The work reported is supported by the National Natural Science Foundation of China (NSFC) (Grant No. 11023001), and computation is mainly supported by the Supercomputing Center of Chinese Academy of Sciences (SCCAS) and Shanghai Supercomputer Center (SSC). N.P. has been sup- ported by the European Research Council under the European Union's Seventh Framework Programme (FP7/20072013)/ERC Grant agreement No. [279985] (ERC StG Ideas Award to N.M.P. on "Bio-inspired hierarchical super nanomaterials (BIHSNAM)").

${ }^{1}$ K. S. Novoselov, A. K. Geim, S. V. Morozov, D. Jiang, Y. Zhang, S. V. Dubonos, I. V. Grigorieva, and A. A. Firsov, Science 306, 666-669 (2004).

${ }^{2}$ J. C. Meyer, A. K. Germ, M. I. Katsnelson, K. S. Novoselov, T. J. Booth, and S. Roth, Nature 446, 60-63 (2007).

${ }^{3}$ K. S. Novoselov, A. K. Geim, S. V. Morozov, D. Jiang, M. I. Katsnelson, I. V Grigorieva, S. V. Dubonos, and A. A. Firsov, Nature 438, 197-200 (2005).

${ }^{4}$ A. K. Geim and K. S. Novoselov, Nature Mater. 6, 183-191 (2007).

${ }^{5}$ C. Lee, X. Wei, J. W. Kysar, and J. Hone, Science 321, 385-388 (2008).

${ }^{6}$ L. A. Ponomarenko, F. Schedin, M. I. Katsnelson, R. Yang, E. W. Hill, K. S. Novoselov, and A. K. Geim, Science 320, 356-358 (2008).

${ }^{7}$ H. C. Ko, M. P. Stoykovich, J. Z. Song, V. Malyarchuk, W. M. Choi, C. J. Yu, J. B. Geddes III, J. L. Xiao, S. D. Wang, Y. Huang, and J. A. Rogers, Nature 454, 748 (2008).

${ }^{8} \mathrm{Z}$. Xu and M. J. Buehler, ACS Nano 4, 3869 (2010).

${ }^{9}$ X. H. Shi, Y. Cheng, N. M. Pugno, and H. J. Gao, Small 6, 739 (2010).

${ }^{10}$ E. Cerda and L. Mahadevan, Phys. Rev. Lett. 90, 074302 (2003).

${ }^{11}$ W. Bao, F. Miao, Z. Chen, H. Zhang, W. Jiang, C. Dames, and C. Lau, Nat. Nanotechnol. 4, 562 (2009).

${ }^{12}$ Y. Huang, J. Wu, and K. C. Hwang, Phys. Rev. B 74, 245413 (2006).

${ }^{13}$ Q. Lu and R. Huang, Int. J. Appl. Mech. 1, 443 (2009).

${ }^{14}$ Z. Xu, J. Comput. Theor. Nanosci. 6, 625 (2009).

${ }^{15}$ H. Zhao, K. Min, and N. R. Aluru, Nano Lett. 9, 3012 (2009).

${ }^{16}$ F. Liu, P. B. Ming, and J. Li, Phys. Rev. B 76, 064120 (2007).

${ }^{17}$ G. Gui, J. Li, and J. X. Zhong, Phys. Rev. B 78, 075435 (2008).

${ }^{18}$ R. Faccio, P. A. Denis, H. Pardo, C. Goyenola, and A. W. Mombrú, J. Phys.: Condens. Matter 21, 285304 (2009).

${ }^{19}$ Y. W. Gao, P. Hao, Physica E (Amsterdam) 41, 1561 (2009).

${ }^{20}$ K. Xue and Z. Xu, Appl. Phys. Lett. 96, 063103 (2010).

${ }^{21}$ E. Hernánddez, C. Goze, P. Bernier, and A. Rubio, Phys. Rev. Lett. 80, 4502-4505 (1998).

${ }^{22}$ Q. Lu, M. Arroyo, and R. Huang, J. Phys. D: Appl. Phys. 42, 102002 (2009).

${ }^{23}$ Z. C. Tu and Z. C. Ou-Yang, Phys. Rev. B 65, 233407 (2002).

${ }^{24}$ J. Tersoff, Phys. Rev. B 37, 6991 (1988).

${ }^{25}$ M. Arroyo and T. Belytschko, Phys. Rev. B 69, 115415 (2004).

${ }^{26}$ D. Sanchez-Portal, E. Artacho, J. M. Soler, A. Rubio, and P. Ordejon, Phys. Rev. B 59, 12678 (1999).

${ }^{27}$ K. N. Kudin, G. E. Scuseria, and B. I. Yakobson, Phys. Rev. B 64, 235406 (2001).

${ }^{28}$ D. B. Zhang, E. Akatyeva, and T. Dumitrică, Phys. Rev. Lett. 106, 255503 (2011).

${ }^{29}$ G. Kresse and J. Furthmüller, Phys. Rev. B 54, 11169 (1996).

${ }^{30}$ G. Kresse and J. Furthmüller, Comput. Mater. Sci. 6, 15 (1996).

${ }^{31}$ P. E. Blochl, Phys. Rev. B 50, 17953 (1994).

${ }^{32}$ R. Saito, M. Fujita, G. Dresselhaus, and M. S. Dresselhaus, Physical Properties of Carbon Nanotubes (Imperial College London, London, 1998).

${ }^{33}$ G. Grosso and C. Piermarocchi, Phys. Rev. B 51, 16772 (1995).

${ }^{34}$ X. H. Shi, N. M. Pugno, and H. J. Gao, J. Comput. Theor. Nanosci. 7, 517 (2010).

${ }^{35}$ S. Grimme, J. Comput. Chem. 27, 1787 (2006). 